\title{
Analysis of Ligand-Receptor Association and Intermediate Transfer Rates in Multi-enzyme Nanostructures with All-Atom Brownian Dynamics Simulations
}

Christopher C. Roberts, Chia-en A. Chang

Department of Chemistry, University of California, Riverside, California, 92521, United States

\section{Supporting Information}

\section{Diffusion Coefficients and Stokes Radii}

In this manuscript, the radii of gyration of the small molecular substrates were used to estimate the diffusion coefficients of each substrate using the Stokes-Einstein equation. We utilized the radius of gyration to approximate the hydrodynamic radius because in the cases of hydrogen peroxide and tetramethylbenzidine, the calculated values approximately equal those found from experimental values or through analysis of molecular dynamics simulations of the solute in TIP3P water. The Stokes radius of hydrogen peroxide calculated from an experimentally determined diffusion coefficient $\left(\mathrm{D}=0.14 \AA^{2} / \mathrm{ps}^{92-94}\right)$ gives a value of $1.5 \AA$. The radius of gyration of hydrogen peroxide for the conformation we used gives a value of $1.2 \AA$. The hydrodynamic radius can also be approximated according to the following Equation S1:

$$
\frac{1}{R_{H}}=\frac{1}{N^{2}} \sum \frac{1}{r_{i j}}
$$

where $N$ is the number of particles in the molecule or molecular model, and $r_{i j}$ is the distance between particles $i$ and $j$. Using this formulation gives a much larger approximated radius for hydrogen peroxide of $4.3 \AA$. Likewise, calculating the diffusion coefficient of TMB $(\mathrm{D} \sim=0.06$ $\AA^{2} / \mathrm{ps}$ ) in a TIP3PBOX (AMBER GAFF+FF14SB) reveals a Stokes radius of $3.6 \AA$. The radius of gyration of TMB is $4.1 \AA$ while the hydrodynamic radius is $7.9 \AA$. Because of the close agreement between the radius of gyration and Stokes radius calculated from experiments or simulations, and for the sake of consistency, we have used the radius of gyration as the basis for the diffusion coefficient calculations in the manuscript.

The radii of gyration for the receptors used in this work are as presented in Table S1 for reference. These values were calculated by GeomBD2 at simulation run time, and were included in the relative diffusion coefficients for the appropriate substrate. 


\begin{tabular}{|l|l|}
\hline Receptor System & Radius of Gyration $(\AA)$ \\
\hline HRP & 19.2 \\
\hline HRP+DNA1 & 31.4 \\
\hline HRP+DNA2 & 30.5 \\
\hline HRP+GOx Artificially Colocalized & 52.4 \\
\hline HRP+GOx in Planar DNA scaffold & 286.7 \\
\hline HRP+GOX on Tubular DNA scaffold & 249.7 \\
\hline HIV protease & 17.9 \\
\hline
\end{tabular}

Table S1. Calculated radii of gyration for the receptor systems used in the manuscript. These values were included in the relative diffusion coefficients of substrate. The presented systems include Horseradish peroxidase (HRP), the DNA conjugated HRP in position 1 (HRP+DNA1), the DNA conjugated HRP in position 2 (HRP+DNA2), Glucose oxidase (GOx), and the Human Immunodeficiency Virus protease enzyme (HIV protease). 


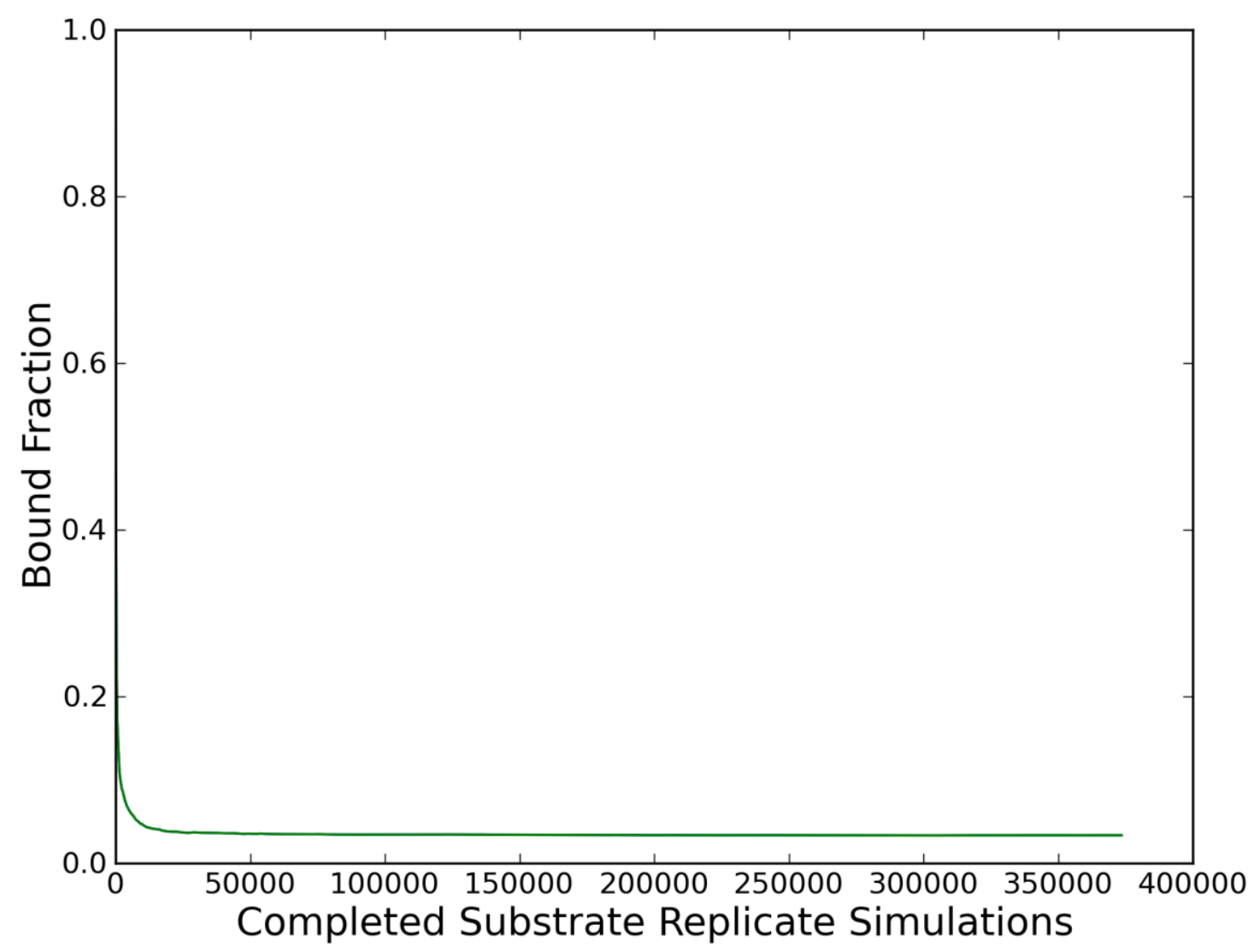

Figure S1. Example plot of the bound fraction as it converges with increasing number of completed replicate simulations. This data is derived from the association of $\mathrm{H}_{2} \mathrm{O}_{2}$ associating with the HRP enzyme. This plot was created with the plot_convergence.py GeomBD2 analysis script. 